\title{
Effects of mean shear on the local turbulent entrainment process
}

\section{Journal Article}

\section{Author(s):}

Wolf, Marc; Holzner, Markus; Lüthi, Beat; Krug, Dominik; Kinzelbach, Wolfgang (D); Tsinober, Arkady

Publication date:

2013-09-25

Permanent link:

https://doi.org/10.3929/ethz-b-000072588

Rights / license:

In Copyright - Non-Commercial Use Permitted

\section{Originally published in:}

Journal of Fluid Mechanics 731, https://doi.org/10.1017/jfm.2013.365 


\title{
Effects of mean shear on the local turbulent entrainment process
}

\author{
Marc Wolf $^{1} \dagger$, M. Holzner ${ }^{1}$, B. Lüthi ${ }^{1}$, D. Krug ${ }^{1}$, W. Kinzelbach ${ }^{1}$ \\ and A. Tsinober ${ }^{2}$ \\ ${ }^{1}$ Institute of Environmental Engineering, ETH Zurich, 8093 Zurich, Switzerland \\ ${ }^{2}$ School of Mechanical Engineering, Faculty of Engineering, Tel Aviv University, Tel Aviv 69978, Israel
}

(Received 5 February 2013; revised 12 June 2013; accepted 12 July 2013; first published online 14 August 2013)

We report on effects of mean shear on the turbulent entrainment process, focusing in particular on their relation to small-scale processes in the proximity of the turbulent/non-turbulent interface (TNTI). Three-dimensional particle tracking velocimetry (3D-PTV) measurements of an axisymmetric jet are compared to data from a direct numerical simulation (DNS) of a zero-mean-shear (ZMS) flow. First, conditional statistics relative to the interface position are investigated in a pseudoEulerian view (i.e. in a fixed frame relative to the interface position) and in a Lagrangian view. We find that in a pseudo-Eulerian frame of reference, both vorticity fluctuations and mean shear contribute to the vorticity jump at the boundary between irrotational and turbulent regions. In contrast, the Lagrangian evolution of enstrophy along trajectories crossing the entrainment interface is almost exclusively dominated by vorticity fluctuations, at least during the first Kolmogorov time scales after passing the interface. A mapping between distance to the instantaneous interface versus conditional time along the trajectory shows that entraining particles remain initially close to the TNTI and therefore attain lower average enstrophy values. The ratio between the rate of change of enstrophy in the two frames of references defines the local entrainment velocity $v_{n}=-\left(\mathrm{D} \omega^{2} / \mathrm{D} t\right) /\left(\partial \omega^{2} / \partial \hat{x}_{n}\right)$, where $\omega^{2}$ is enstrophy and $\hat{x}_{n}$ is the coordinate normal to the TNTI. The quantity $v_{n}$ is decomposed into mean and fluctuating components and it is found that mean shear enhances the local entrainment velocity via inviscid and viscous effects. Further, the analysis substantiates that for all investigated flow configurations the local entrainment velocity depends considerably on the geometrical shape of the interface. Depending on the surface shape, different small-scale mechanisms are dominant for the local entrainment process, i.e. viscous effects for convex shapes and vortex stretching for concave shapes, looking from the turbulent region towards the convoluted boundary. Moreover, turbulent fluctuations display a stronger dependence on the shape of the interface than mean shear effects.

Key words: shear layers, turbulent flows, wakes/jets

\section{Introduction}

Transport of irrotational fluid particles across the free stream boundary between turbulent and non-turbulent flow regions is an important aspect of many engineering 
and geophysical flows, including jets, mixing layers, wakes, and boundary layers. This process, commonly referred to as turbulent entrainment, occurs over a strongly convoluted interface and directly affects dynamics and mixing of turbulent flows, thereby controlling transport rates of energy, heat, and mass (Townsend 1976). Thus, the study of the entrainment process is not only relevant from a basic point of view, but also an important part of physical fundamentals of a great variety of technological processes and their control (Hunt et al. 2011). Despite its importance, there are still many open questions that significantly hinder fundamental understanding, proper parametrization, and accurate modelling (Hunt, Eames \& Westerweel 2006; da Silva 2009). As described in Ferrey \& Aupoix (2006), standard turbulence models applied in engineering problems depend upon small levels of eddy viscosity outside of the turbulent region, which do not reflect the physical behaviour near the turbulent/nonturbulent interface (TNTI) (see also Westerweel 2005). A number of recent studies have provided new insights into the phenomenon of turbulent entrainment, improving the conceptual understanding of the process (see e.g. Holzner et al. 2008; da Silva 2009; Westerweel et al. 2009; Philip \& Marusic 2012, and references therein).

A long-standing question in this context is the interaction of small- and large-scale eddy motions, both influencing the turbulent entrainment process. On one hand, it is widely accepted that the main mechanism by which non-turbulent fluid becomes turbulent as it crosses the interface is inferred to involve viscous diffusion (see e.g. Corrsin \& Kistler 1954; Dahm \& Dimotakis 1990; Bisset, Hunt \& Rogers 2002; Westerweel 2005; Holzner et al. 2008; Tsinober 2009; da Silva, dos Reis \& Pereira 2011). This is believed to be one of the reasons why the interface appears sharp. On the other hand, it is also well known that at large Reynolds numbers the entrainment rate and the propagation velocity of the interface relative to the fluid are independent of viscosity (see e.g. Ricou \& Spalding 1961; Coles 1962; Hinze 1975; Tritton 1988; Govindarajan 2004; Hunt et al. 2006). From this it follows that the slow process of diffusion into the ambient fluid must somehow be amplified. Townsend (1976) explained that interacting velocity fields of eddies of all sizes, from viscous eddies to the energy-containing eddies, produce a strongly contorted interface, resulting in a higher overall entrainment. In other words, entrainment can be understood as a diffusion process across a strongly convoluted interface, where presumably the large surface area causes a cumulative effect that cancels out the viscosity dependence. As outlined by Sreenivasan, Ramshankar \& Meneveau (1989), the global entrainment flux $Q$ can either be determined by the mean entrainment velocity $u_{e}$ times a mean projected area $A_{0}$ or by a small-scale local entrainment velocity $v_{n}$ together with the strongly convoluted instantaneous turbulent/non-turbulent interface area, so that $Q=u_{e} A_{0}=\int v_{n} \mathrm{~d} A$. Hence, local entrainment velocity and instantaneous interface area represent key parameters of the entrainment process linking small-scale processes of the flow to the global entrainment rate. Holzner \& Lüthi (2011) recently showed that the instantaneous interface area is strongly convoluted to account for a large entrainment flux with a small characteristic velocity comparable to the Kolmogorov velocity, $v_{n} \sim u_{\eta}$.

In an early experimental and theoretical study, Corrsin \& Kistler (1954) postulated that the local entrainment velocity in a free shear flow depends upon a shear force that is fed from small-scale fluctuations as well as mean shear. Recent findings by da Silva \& Taveira (2010) support the concept that mean shear has an influence on the entrainment process. In particular, they show that the thickness of the turbulent/nonturbulent interface is related to large vorticity structures whose lifetimes depend on Reynolds number and mean shear. Furthermore, a theoretical and experimental work 
by Westerweel et al. (2009) demonstrates that the propagation velocity of the turbulent front in a jet flow has a contribution from turbulent fluctuations and from a jump in the mean velocity across the turbulent boundary, which is essentially a mean shear.

The goal of the presented work is to investigate effects of mean shear on the local entrainment velocity. We compare experimental data of an axisymmetric jet to results from a DNS of a flow with zero mean shear (ZMS). First, conditional enstrophy profiles relative to the interface position are characterized in a pseudo-Eulerian view (i.e. in a fixed frame relative to the interface position) and in a Lagrangian view. Secondly, extending the analysis in Wolf et al. (2012), the local entrainment velocity $v_{n}$ is investigated with respect to small-scale mechanisms such as vortex stretching and viscous effects, as well as to the geometry of the interface. Effects of mean shear and universal features of the entrainment process are discussed. The intention of the study is to highlight some interesting effects of mean shear on the entrainment process.

The paper is organized as follows. In $\S 2$ we describe the experimental jet measurements, give information about direct numerical simulation (DNS), and discuss the detection procedure of the entrainment interface as well as conditional sampling of the flow data. In $\S 3$ we assess conditional statistics in the pseudo-Eulerian and Lagrangian frames of reference and discuss effects of mean shear on the local entrainment velocity. Finally, a summary and some suggestions for future studies are given in $\S 4$.

\section{Method}

In this section we describe the experimental jet measurements and give information about DNS of the ZMS flow. Both methods have been described in detail in Wolf et al. (2012) (experiment) and in Holzner et al. (2008) (simulation) and only the main features are presented in the following. Furthermore, the detection of the entrainment interface, conditional sampling of flow data, and a mathematical description of the local entrainment velocity are briefly summarized.

\subsection{Experimental jet measurements}

Experimental data of an axisymmetric jet flow was obtained from three-dimensional particle tracking velocimetry (3D-PTV) measurements in a closed-loop jet facility using water as the working fluid. An interrogation volume at the boundary between the turbulent jet and irrotational ambient fluid was investigated, and instantaneous distributions of velocity and velocity gradients were determined in the volume.

The test section of the experimental setup is illustrated in figure 1(a). A turbulent jet emerges from the end of a thin pipe (inner diameter $d=3 \mathrm{~mm}$, length $l=240 \mathrm{~mm}$ ) into a cylindrical glass tank of diameter $D=300 \mathrm{~mm}$ and length $L=2000 \mathrm{~mm}$, which contains quiescent ambient fluid. The fluid in the cylinder and the jet is water at room temperature. The jet pipe and cylindrical water tank are each connected to a constanthead reservoir. The two reservoirs are positioned at different heights producing a constant hydrostatic pressure difference, which ensures a steady flow rate (constanthead reservoirs are not shown in the sketch). In order to avoid image distortions caused by the difference in index of refraction between the inside and outside of the cylindrical water tank, a rectangular glass box, also filled with water, is fitted around the cylinder. As described in Wolf et al. (2012), the experimental facility was thoroughly tested and validated using two-dimensional PTV measurements at the centre plane of the axisymmetric jet. It was shown that a self-preserving turbulent jet is established for a downstream position larger than $30 x / d$ and that velocity decay 

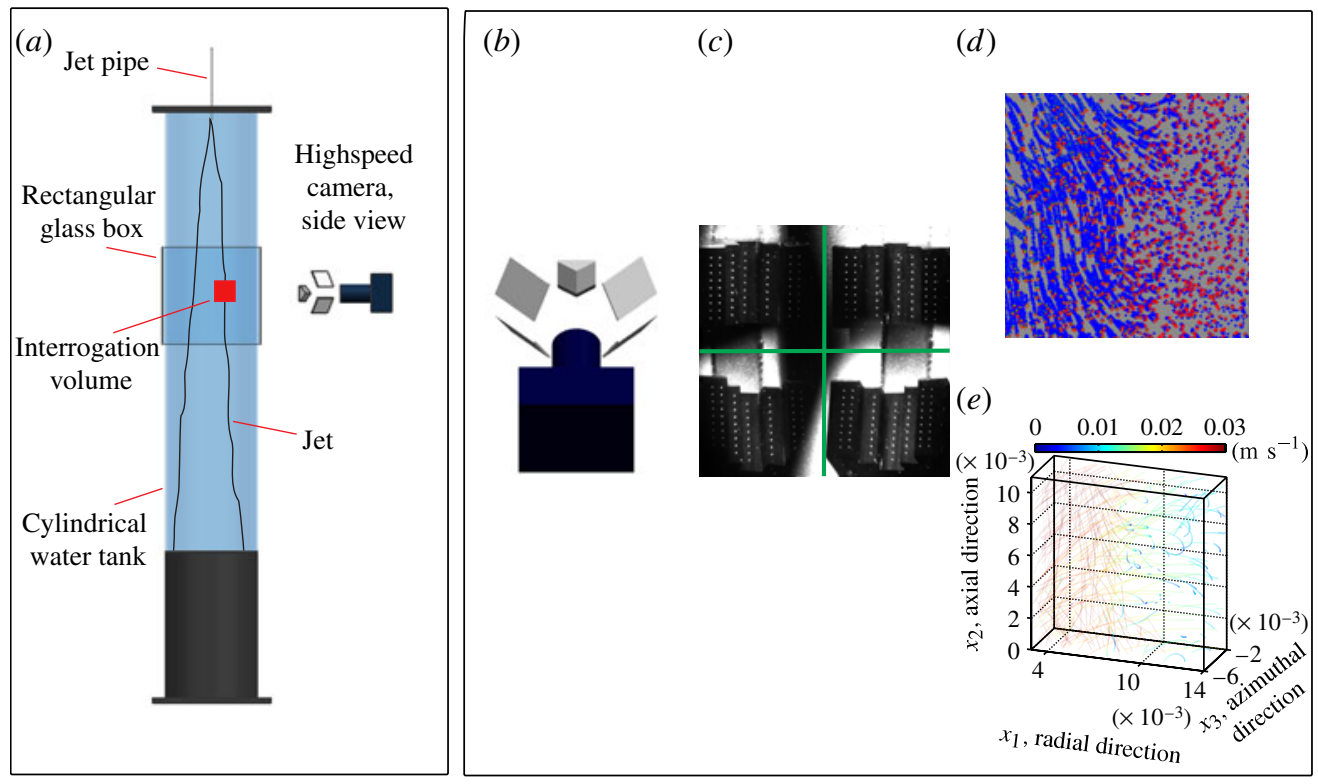

FIGURE 1. (Colour online) Sketch of experimental setup and PTV processing. (a) Sketch of test section. (b) High-speed camera with image splitter, top view. (c) Multi-camera view example: target block. $(d)$ Two-dimensional trajectories as obtained by one camera view. (e) Three-dimensional trajectories colour-coded with the velocity magnitude.

constant $\mathrm{C} 1=U_{c} *\left(x-x_{0}\right) / U_{0} * d=6.1$, and spreading rate $\mathrm{C} 2=\mathrm{d} b_{0.5} / \mathrm{d} x=0.09$, where $U_{0}$ is the average exit velocity of the jet pipe, $U_{c}$ the mean centreline velocity, and $b_{0.5}$ the velocity half-width, lie in the range reported in the literature (see e.g. Wygnanski \& Fiedler 1969; Hussein, Capp \& George 1994; Xu \& Antonia 2002).

Relevant processing steps of the 3D-PTV measurements are shown in figure $1(b-e)$. A classical PTV system uses four synchronized cameras ensuring a robust stereoscopic detection of flow tracers within the established seeding limit of $\sim 0.05$ particles per pixel (Maas, Gruen \& Papantoniou 1993). For our measurements, we employ one single camera in combination with a four-way image splitter to mimic the classical four-camera setup, as illustrated in figure $1(b)$ and described in detail in Hoyer et al. (2005). In this way, all four views of the interrogation volume are recorded onto a single image simultaneously. An image example showing the target block used for the calibration of the system is depicted in figure 1(c). In a first processing step, a spatiotemporal tracking algorithm (Willneff \& Gruen 2002) is used to determine particle trajectories in image space for every camera view. An example of two-dimensional trajectories at the jet interface is shown in figure $1(d)$. Subsequently, three-dimensional trajectories are constructed in object space, and Lagrangian quantities such as velocity and acceleration are calculated along the trajectory. The resulting trajectories in the interrogation volume investigated are depicted in figure 1(e). The three-dimensional trajectories are colour-coded with the velocity magnitude. In our measurements, the average interparticle distance was of the order of the smallest length scales of the flow, so that we also obtained spatial derivatives of velocity and acceleration; for details on the calculation procedure see Lüthi, Tsinober \& Kinzelbach (2005). 


$\begin{array}{lcccc} & \text { Zero mean shear } & \text { Jet } \operatorname{Re} 2000 & \text { Jet } R e \text { S000 } & \text { Jet } R e 8000 \\ \eta & 1.5 \Delta x & 0.46 \mathrm{~mm} & 0.33 \mathrm{~mm} & 0.26 \mathrm{~mm} \\ \tau_{\eta} & 250 \Delta t & 0.21 \mathrm{~s} & 0.11 \mathrm{~s} & 0.07 \mathrm{~s} \\ \operatorname{Re}_{\lambda} & 50 & 60 & 80 & 100\end{array}$

TABLE 1. Kolmogorov scales and $R e_{\lambda}$ for the three jet experiments and the DNS of the ZMS flow. $\Delta x$ and $\Delta t$ represent the grid spacing and time step of the simulation.

For the 3D-PTV measurements investigating the jet interface, we used a Photron SA 5 camera $\left(1024 \times 1024\right.$ pixels $^{2}$, object lens of $\left.200 \mathrm{~mm}\right)$ in combination with the above-mentioned image splitter. The camera was focused on an interrogation volume of $11 \mathrm{~mm} \times 11 \mathrm{~mm} \times 4 \mathrm{~mm}$, as indicated by a small square in figure 1(a). Using a non-cubic observation volume reduces the number of ambiguities in the stereoscopic detection procedure, which improves the establishment of correspondences in the tracking process. This means that a higher number of particles can be tracked in space and time, resulting in a better spatial resolution. The measurement volume was situated $92 x / d-96 x / d$ downstream of the nozzle exit. Illumination of the interrogation volume was provided by a continuous $15 \mathrm{~W}$ argon ion laser. The laser beam was expanded through two spherical lenses. The jet flow and ambient fluid were seeded with neutrally buoyant polystyrene tracer particles with an average diameter of $45 \mu \mathrm{m}$. In our recordings, $\sim 200$ particles could be tracked on average in the interrogation volume, yielding an average particle distance of $\sim 1.3 \mathrm{~mm}$. The particle position accuracy of the measurements was $0.1 \mathrm{~mm}$ for the $x_{1}$ and $x_{2}$ components and $0.3 \mathrm{~mm}$ for the $x_{3}$ component respectively, where $x_{1}, x_{2}$ and $x_{3}$ correspond to the radial, axial and azimuthal jet axes. Measurements for three different jet velocities $U_{0}(0.67,1.67$, $\left.2.67 \mathrm{~m} \mathrm{~s}^{-1}\right)$ were conducted, implying jet Reynolds numbers, $R e=\left(U_{0} * d\right) / \nu$, of 2000 , 5000,8000 respectively, where $v$ is the kinematic viscosity of the fluid. Table 1 lists Kolmogorov scales for length, $\eta$, and time, $\tau_{\eta}$, as well as the estimated Taylor Reynolds number, $R e_{\lambda}=\sqrt{15}(L / \eta)^{2 / 3}$, with $L$ being the integral scale approximated from the velocity half-width. Kolmogorov scales are determined from the average dissipation rate in the turbulent region, $\epsilon=2 v\left\langle s_{i j} s_{i j}\right\rangle$. Recordings were taken at a frame rate of $500 \mathrm{~Hz}$ for the cases $\operatorname{Re} 2000$ and $R e 5000$ and $1000 \mathrm{~Hz}$ for the case $\operatorname{Re} 8000$, corresponding to a temporal resolution faster than $1 / 10 \tau_{\eta}$ in each case. The spatial resolution yields $2 \eta, 4 \eta$ and $5 \eta$ for $\operatorname{Re} 2000,5000$ and 8000 respectively.

A detailed accuracy analysis has been reported in Wolf et al. (2012) for the case of $R e$ 5000, assessing the quality of the performed 3D-PTV measurements. It was shown that the random error in the determination of velocity and acceleration derivatives is $\sim 10 \%$, which lies in the range of other PTV and particle image velocimetry (PIV) studies investigating small-scale aspects of turbulent flows (see e.g. Mullin \& Dahm 2006; Ganapathisubramani, Lakshminarasimhan \& Clemens 2007; Holzner et al. 2009; Liberzon et al. 2012). Random errors for the cases of $R e 2000$ and $R e 8000$ are comparable to the ones obtained for $R e$ 5000. As an example of the applied accuracy checks, we show in figure 2 the joint probability density functions (joint p.d.f.s) of the total velocity derivative versus local and convective acceleration. It can be seen that the data points are distributed along the diagonal for all three cases, i.e. no systematic error is apparent. Furthermore, the aspect ratios of the distributions, which give an indication of the random error, are similar for the three Reynolds numbers. Correlation factors of the checks lie in the range of 0.84-0.94 for all three Reynolds numbers. 
(a)

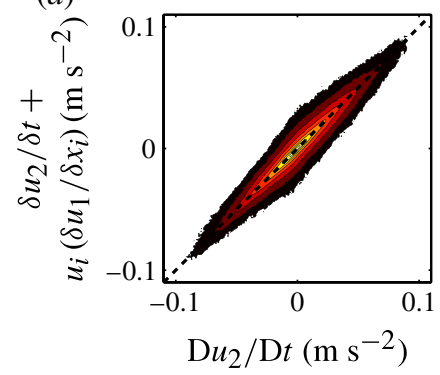

(b)

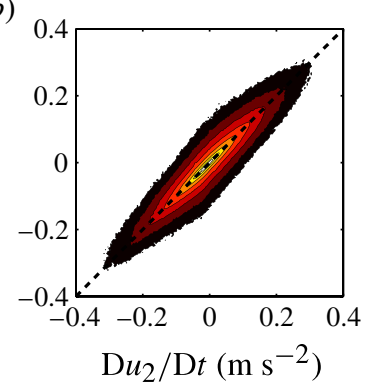

(c)

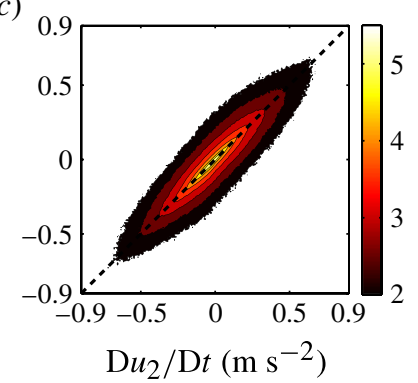

FIgURE 2. (Colour online) Accuracy checks: joint p.d.f.s of total velocity derivative $\mathrm{D} u_{i} / \mathrm{D} t$ versus local and convective acceleration $\partial u_{i} / \partial t+u_{j}\left(\partial u_{i} / \partial x_{j}\right)$, depicted for the velocity component in the $x_{2}$ direction. (a) jet $\operatorname{Re} 2000,(b)$ jet $\operatorname{Re} 5000,(c)$ jet $\operatorname{Re} 8000$.

\subsection{Direct numerical simulation of a flow with $Z M S$}

In addition to the experimental jet data, this study relies upon data from DNS of a ZMS flow. Previous studies conducted by some of the authors have already employed the same numerical data set to examine small-scale features of turbulent entrainment (Holzner et al. 2007, 2008, 2009; Holzner \& Lüthi 2011). In the current study we compare the simulations to the jet experiments described above in order to investigate certain aspects of the influence of mean shear on local entrainment. In summary, the DNS was performed in a box (side lengths $5 L, 3 L, 5 L$ ) of fluid initially at rest. Random (in space and time) velocity perturbations were applied at the boundary $x_{2}=0$, which were generated as follows. For a fixed time and in the discrete set of points, i.e. $x_{1}=k \Delta_{l}, x_{3}=l \Delta_{l}$ ( $k, l$ are integers), each velocity component $u_{i}(i=1,2,3)$ is calculated as $u_{i}=V_{i} \xi$, where $\xi$ is a random number within the interval $[-1,1]$ and $V_{i}$ is a given velocity amplitude. For other times and spatial points $\left(x_{1}, x_{3}\right)$ boundary velocities are obtained by cubic interpolation in time and bilinear interpolation in space. At each time, the three boundary velocity components yield zero average value over the boundary plane. Periodic boundary conditions are applied for the directions $x_{1}$ and $x_{3}$. Shear-free conditions $\delta u_{1} / \delta x_{2}=\delta u_{3} / \delta x_{2}=u_{2}=0$ are set at the boundary $x_{2}=L_{2}$. The Navier-Stokes equations were solved with a finite difference scheme and with time advancement computed by a semi-implicit Runge-Kutta method (Nikitin 2006). The resolution is $256 \times 256 \times 256$ grid points. Kolmogorov scales and Taylor Reynolds numbers for the ZMS flow are depicted in table 1 . The subsequent analysis was carried out for time steps when the advancing turbulent front is about half a box size away from the forcing plane.

\subsection{Interface detection and conditional sampling}

Like Bisset et al. (2002), Holzner et al. (2007), da Silva \& Pereira (2008), and others, we identify the interfacial region between turbulent flow (i.e. jet or ZMS flow) and irrotational ambient flow by using a threshold on enstrophy, $\omega^{2}=\omega \cdot \omega$, where $\omega$ is the vorticity vector. Similar to Holzner et al. (2009) and Wolf et al. (2012), the threshold is set to $\sim 2 \%$ of the mean enstrophy value in the turbulent region. The value is used to determine an enstrophy iso-surface situated in the outer section of the interfacial region between turbulent and irrotational flows. This iso-surface is subsequently tracked in space and time for measurements as well as simulations. Please note that the standard deviation of the measured enstrophy fluctuations in the 

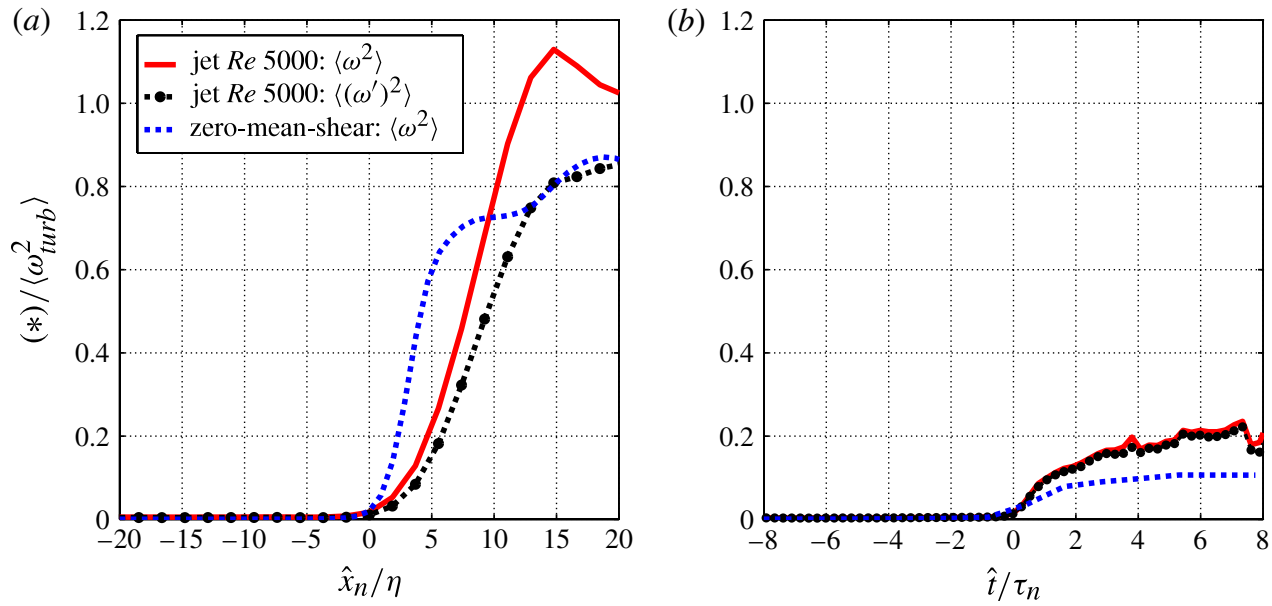

FIGURE 3. (Colour online) (a) Conditional averages of total enstrophy $\left\langle\omega^{2}\right\rangle$ and squared vorticity fluctuations $\left\langle\left(\omega^{\prime}\right)^{2}\right\rangle$ in the normal direction to the interface; Eulerian frame of reference. $(b)$ Conditionally averaged Lagrangian evolutions of total enstrophy $\left\langle\omega^{2}\right\rangle$ and squared vorticity fluctuations $\left\langle\left(\omega^{\prime}\right)^{2}\right\rangle$; Lagrangian frame of reference. Enstrophy values are normalized by its average in the turbulent region $\left\langle\omega_{\text {turb }}^{2}\right\rangle$. Relative distance and time are normalized by Kolmogorov scales. Negative values represent potential flow and positive values turbulent regions, respectively.

ambient flow region, due to measurement noise, are about four times smaller than the chosen thresholds for the three jet cases.

Furthermore, flow properties are analysed relative to the interface and conditional statistics are computed by averaging data at fixed distances to the interface position. We define a new coordinate system $\hat{\boldsymbol{x}}$, where the origin is located at the iso-surface and the normal direction is given as $\hat{x}_{n}$. Negative values of $\hat{x}_{n}$ represent the potential flow region and positive values the turbulent region; see e.g. figure 3(a). Following the approach of Bisset et al. (2002) and Westerweel et al. (2009), we decompose the instantaneous velocity field into

$$
u=U+u^{\prime}
$$

where $U$ is the conditional average relative to the interface, $U=\langle u\rangle$, and $u^{\prime}$ is the corresponding fluctuating component, $\left\langle u^{\prime}\right\rangle=0$. In other words, the brackets $\langle\cdots\rangle$ define the ensemble average for a fixed normal distance to the interface, i.e. for a fixed $\hat{x}_{n}$ value.

\subsection{Local entrainment velocity}

A short discussion of the local entrainment velocity is given in the following to introduce main variables needed for the subsequent analysis. A detailed mathematical derivation of $v_{n}$ as well as its connection to small-scale processes can be found in Pope (1988), Dopazo (2006) (scalar iso-surface), and Holzner \& Lüthi (2011). The evolution of the turbulent/non-turbulent interface results from advection due to the underlying flow field and a movement relative to the fluid caused by turbulent entrainment. From this it follows that the velocity of an iso-surface element, $\boldsymbol{u}^{\boldsymbol{s}}$, can be written as the sum of fluid velocity, $\boldsymbol{u}$, and velocity of the area element relative to the fluid, $\boldsymbol{V}=v_{n} \boldsymbol{n}$, that is, $\boldsymbol{u}^{\boldsymbol{s}}=\boldsymbol{u}+\boldsymbol{V}$, where $\boldsymbol{n}=\nabla \omega^{2} /\left|\nabla \omega^{2}\right|$ the surface normal. 
As shown in Holzner \& Lüthi (2011), one can derive an expression for the relative velocity by looking at the movement of the enstrophy iso-surface in a Lagrangian frame of reference, i.e. 'sitting' on the iso-surface, which leads to

$$
v_{n}=-\frac{\frac{\mathrm{D} \omega^{2}}{\mathrm{D} t}}{\left|\nabla \omega^{2}\right|} .
$$

Please note that $v_{n}$ adopts positive values for an orientation in the direction of the enstrophy gradient. This implies that $v_{n}$ of a surface spreading into the irrotational region is on average negative. With the use of the enstrophy transport equation one can decompose $v_{n}$ into the sum of an inviscid and a viscous contribution:

$$
v_{n}=-\frac{2 \omega_{i} \omega_{j} s_{i j}}{\left|\nabla \omega^{2}\right|}-\frac{2 \nu \omega_{i} \nabla^{2} \omega_{i}}{\left|\nabla \omega^{2}\right|}=v_{n}^{i n v}+v_{n}^{v i s} .
$$

\section{Results}

In the following section, the local entrainment velocity is analysed from different perspectives in order to highlight the effects of mean shear on the entrainment process. First, we address $v_{n}$ by its definition given in (2.2) and discuss in this context conditional enstrophy profiles in a pseudo-Eulerian and a Lagrangian frame of reference. Subsequently, the local entrainment velocity and its components are characterized with respect to the geometrical shape of the interface and to conditional averages of strain and vorticity.

\subsection{Enstrophy jump in a pseudo-Eulerian and in the Lagrangian frames of reference}

In order to get a first insight into the local entrainment process, we investigate conditional enstrophy profiles once relative to the interface location, i.e. conditionally averaged with respect to the moving interface (which would be the pseudo-Eulerian view), and once in a Lagrangian view, where we look at the evolution of $\omega^{2}$ along trajectories crossing the entrainment interface (see figure $3 a, b$ ). As shown in (2.2), the gradients of these curves are directly linked to the local entrainment velocity, since $v_{n}$ is defined by the norm of the enstrophy gradient, $\left|\nabla \omega^{2}\right|=\partial \omega^{2} / \partial \hat{x}_{n}$, and the total derivative of enstrophy, $\mathrm{D} \omega^{2} / \mathrm{D} t$. In the following, enstrophy profiles of the ZMS flow and the jet $R e 5000$ are investigated to highlight governing entrainment effects in the respective frame of reference.

Figure 3(a) depicts conditional averages of enstrophy in the pseudo-Eulerian view. The profiles are normalized by the average enstrophy value in the turbulent region $\left\langle\omega_{\text {turb }}^{2}\right\rangle$, i.e. averaged over all $\hat{x}_{n}>0$, and the distance is normalized by the Kolmogorov length scale $\eta$. The solid red and the dashed blue line depict ensemble averages of the total enstrophy $\left\langle\omega^{2}\right\rangle$ for jet and ZMS respectively. The dot-dashed black curve shows the conditional squared vorticity fluctuations $\left\langle\left(\omega^{\prime}\right)^{2}\right\rangle$ for the jet experiment. In the case of ZMS, the conditionally averaged total enstrophy is equal to the vorticity fluctuations, hence only total enstrophy is depicted. Comparing conditional averages of total enstrophy and of vorticity fluctuations for the jet, one can see that mean shear influences the enstrophy distribution in the interfacial region. Due to the presence of mean enstrophy, the characteristic jump of $\omega^{2}$ after $\hat{x}_{n}=0$ is more pronounced and reaches a maximum of $1.1\left\langle\omega_{\text {turb }}^{2}\right\rangle$ at $\sim 15 \eta$ into the turbulent region. The profile of the squared vorticity fluctuations reaches a value of $0.8\left\langle\omega_{\text {turb }}^{2}\right\rangle$ at $15 \hat{x}_{n} / \eta$, which illustrates that a large part of the sudden increase of total enstrophy is due to small-scale eddy 
motions. Therefore, we note that small scales seem to be governing the entrainment process, which fits the findings by Westerweel et al. (2009), who demonstrated that so-called 'small-scale nibbling processes' dominate the entrainment of irrotational fluid into the turbulent region.

Figure 3(a) also shows that mean enstrophy contributes significantly to the strong increase of total enstrophy in the interfacial region, even producing a local maximum at $15 \hat{x}_{n} / \eta$. The peak in the enstrophy profile is usually attributed to large vortex sheet structures that are situated in the interfacial region (Hunt, Eames \& Westerweel 2008; da Silva \& Taveira 2010). Findings of Westerweel et al. (2009) suggest that this effect can be associated with a jump in the conditional mean velocity. For our analysis we can conclude that in the pseudo-Eulerian frame of reference, both mean and fluctuating components of $\omega^{2}$ seem to influence the conditional enstrophy profile. Interestingly, there is also a difference between vorticity fluctuations of the jet and the ZMS flow. The enstrophy increase for ZMS occurs over a considerably shorter distance than the augmentation of vorticity fluctuations in the jet, which means that the ZMS interface is thinner. This finding is reminiscent of results by da Silva \& Taveira (2010), who show conditional mean profiles of one vorticity component for jets and shear-free isotropic turbulence. They argue that large-scale vorticity structures affect the distance over which the enstrophy increase occurs. Further, they explain that these large-scale vorticity structures have a longer lifetime in jets, therefore exhibiting a higher probability of influencing the entrainment process, which might be a possible explanation for the observed difference.

The Lagrangian evolution of enstrophy along trajectories crossing the entrainment interface is shown in figure $3(b)$. All trajectories are centred at time $\hat{t}$, when the fixed threshold of $\omega^{2}$ is exceeded for the first time. For the statistical analysis, all trajectories are ensemble-averaged, conditioned on $\hat{t}$. Please note that we only take into account particles that cross the interface from the irrotational ambient to the turbulent region. Detraining particles are discarded from the statistics. When particles reach the entrainment interface, their enstrophy level increases on average quite rapidly for about one $\tau_{\eta}$ and grows more gradually inside the turbulent region. This behaviour is observed for jets as well as for ZMS. In contrast to the pseudo-Eulerian view, the temporal enstrophy gradient of the ZMS appears to be slightly smaller than for the jet flow. A stronger spatial gradient in the normal direction in combination with a smaller gradient in the Lagrangian description suggests that the local entrainment velocity attains lower average values for ZMS compared to the jet, which will be further investigated in the subsequent sections. Interestingly, the enstrophy level of entraining particles in the jet flow results almost exclusively from vorticity fluctuations. This means that from a Lagrangian perspective small-scale eddy motions appear to govern the entrainment process, with only a little influence from mean shear. Moreover, one can see that within the first $8 \tau_{\eta}$ after crossing the entrainment interface, entraining particles reach considerably smaller enstrophy levels compared to levels seen in the turbulent region of the pseudo-Eulerian reference frame. Since vorticity is a Galilean invariant, one would expect similar enstrophy levels to be obtained for both frames of reference. One possible reason for the difference is that the ensemble average in the Lagrangian analysis comprises only particles that recently crossed the entrainment interface, whereas in the pseudo-Eulerian view (figure $3 a$ ) the ensemble average consists of all particles present, i.e. of recently entrained particles and particles that have originally been in the turbulent region. A second possible reason is that within 


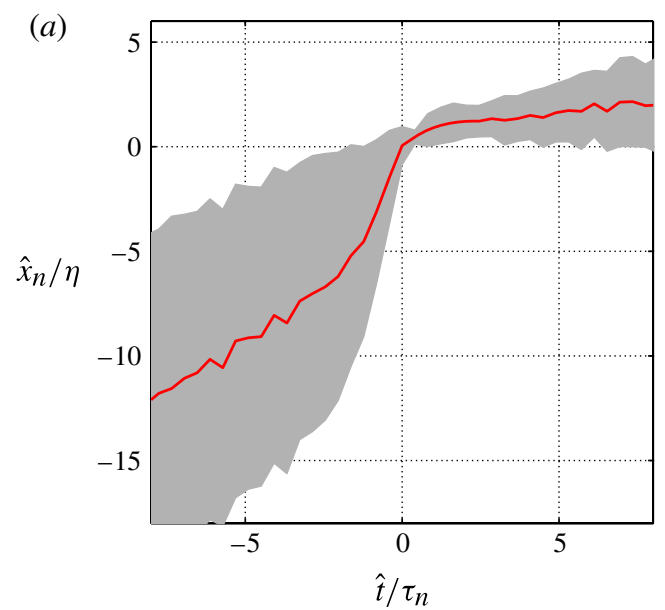

(b)

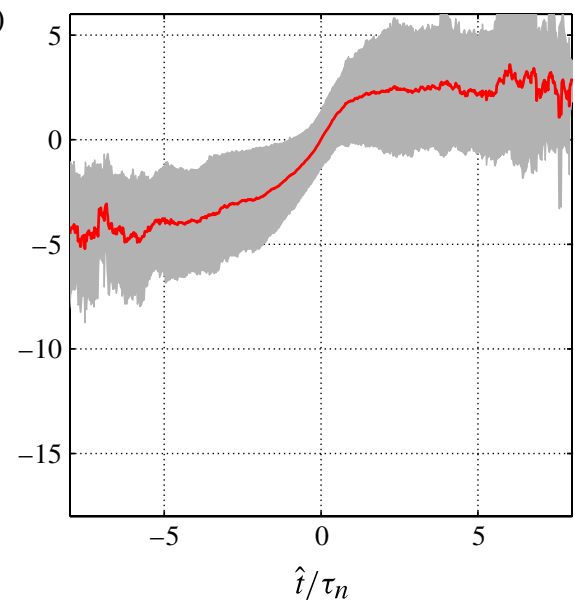

FIGURE 4. (Colour online) Mapping between normal distance to the instantaneous interface $\left(\hat{x}_{n}\right)$ versus conditional time along the trajectory $(\hat{t})$. Values are normalized with the respective Kolmogorov scales: (a) ZMS; (b) jet $\operatorname{Re} 5000$.

the given time span, newly entrained fluid particles may not move on average very deeply into the turbulent region, which is analysed in the subsequent paragraph.

In order to investigate how far entrained particles move away from the interface into the turbulent flow region, figure 4 maps normal distance to the instantaneous interface $\left(\hat{x}_{n}\right)$ versus conditional time along the trajectory $(\hat{t})$. The mapping is presented for $(a)$ ZMS and $(b)$ jet $R e$ 5000, respectively. The red line represents the ensemble average and the shaded grey area corresponds to the standard deviation. As entraining particles cross the turbulent/non-turbulent interface, i.e. the origin, they seem to decelerate quickly and maintain a short, almost constant distance to the interfacial region for several Kolmogorov times after crossing the interface. This is consistent with the lower conditional enstrophy value seen in figure $3(b)$. After a time period of $\sim 5 \tau_{\eta}$, entrained particles are on average no more than $\sim 2 \eta$ away from the interface. Consequently, their mean enstrophy level seen in figure $3(b)$ fits the value in the pseudo-Eulerian frame of reference depicted in figure 3(a). In summary, it is observed that entraining particles initially stay close to the entrainment interface and therefore develop only lower average enstrophy values. This behaviour is apparent both for ZMS and jet. Differences between the flow types in the irrotational flow region, i.e. for negative $\hat{x}_{n}$ and $\hat{t}$ values, might result from tracking limitations in the PTV measurements. For the jet experiments, trajectory lengths were limited to 500 time steps, which corresponds to $10 \tau_{\eta}$. Further, the interrogation domain was also limited to a small volume around the interfacial region. Hence, entraining particles were only detected when they were in the proximity of the entrainment interface. These constraints do not exist for the DNS of the zero mean shear flow, therefore we believe that the mapping for the ZMS is more accurate, especially for the mapping before the crossing of the interface. Nevertheless, both flows show a similar behaviour for positive $\hat{x}_{n}$ and $\hat{t}$ values.

\subsection{Local entrainment velocity and its components in the normal direction}

Figure 5 shows conditional profiles of $v_{n}, v_{n}^{i n v}$ and $v_{n}^{v i s}$ relative to the interface for jet Re 5000. We observe that the local entrainment velocity as well as its components 


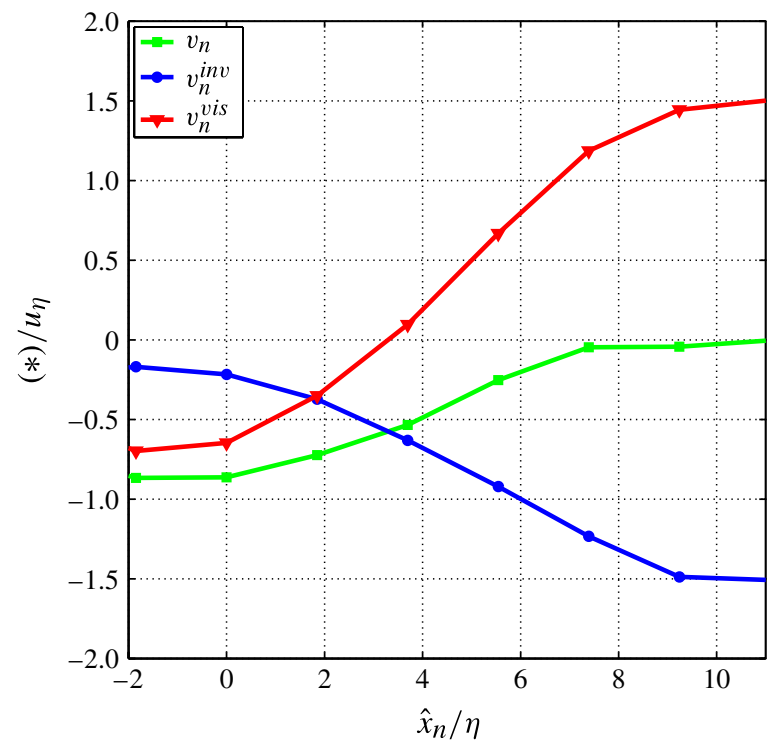

FIGURE 5. (Colour online) Profiles of $v_{n}, v_{n}^{i n v}$ and $v_{n}^{v i s}$ in the normal direction, jet $\operatorname{Re} 5000$.

depend on the distance to the interface. Close to the interface, both inviscid and viscous entrainment velocity drive the outward spreading of the turbulent front. Further inside the turbulent region, $v_{n}^{\text {inv }}$ tries to steepen the enstrophy gradient, whereas $v_{n}^{v i s}$ counteracts the process causing a reduction of the gradient. Sufficiently deep into the turbulent flow $\left(\hat{x}_{n} / \eta>9\right)$, the two processes are in balance and the local entrainment velocity becomes zero. Clearly the choice of the enstrophy threshold influences the position of the origin in this plot and affects the relative balance between viscous and inviscid effects. We want to investigate what happens with $v_{n}^{\text {inv }}$ and $v_{n}^{v i s}$ at the outer region of the TNTI, where viscous effects are known to be dominating on average (Corrsin \& Kistler 1954; Holzner \& Lüthi 2011). We have therefore selected a fairly low threshold value ( $\sim 2 \%$ of the mean enstrophy value in the turbulent region), where viscous effects dominate, yet inviscid effects start to contribute. Furthermore, for a comparison of different flows the respective enstrophy threshold has to be chosen in such a way that inviscid and viscous components of $v_{n}$ show comparable characteristics at the interface for all investigated cases. Only then can resulting differences in the entrainment process for the different configurations be attributed to the character of the flow. For the subsequent analysis, enstrophy thresholds were selected with respect to the condition that ratios of $v_{n}^{\text {inv }} / v_{n}$ and $v_{n}^{v i s} / v_{n}$ yield values of $\sim 0.4$ and 0.6 for all four flow configurations. Exact ratios are depicted in table 2 .

\subsection{Local entrainment velocity conditioned on surface shapes}

In the following section, we discuss the dependence of the local entrainment velocity on the geometrical shape of the instantaneous entrainment interface. The authors have already shown in Wolf et al. (2012) that for a jet flow with a Reynolds number of 5000, the local entrainment velocity changes considerably depending on the local shape of the interface. In the current study, it is investigated how the geometrical dependence of $v_{n}$ changes for different flow types. Figure 6(a) shows a snapshot of an enstrophy iso-surface representing the instantaneous TNTI of jet $R e$ 5000. The 
(a)

$$
\left(\mathrm{s}^{-2}\right)
$$

$\begin{array}{llllllll}10 & 20 & 30 & 40 & 50 & 60 & 70 & 80\end{array}$

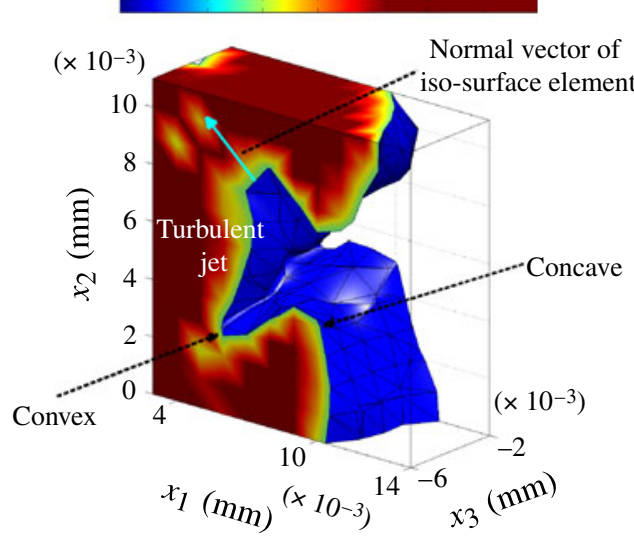

(b)

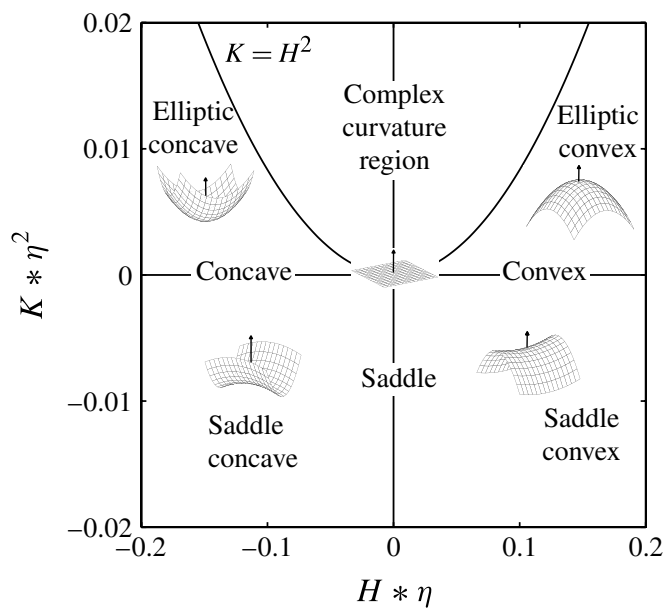

FIgURE 6. (Colour online) (a) Snapshot of enstrophy iso-surface representing the instantaneous entrainment interface of jet $R e$ 5000. The turbulent region is indicated by end-caps colour-coded with the $\hat{\omega}^{2}$ value, adapted from Wolf et al. (2012). (b) Basic surface types depending on Gaussian and mean curvatures $K$ and $H$, adapted from Dopazo, Martin \& Hierro (2007).

turbulent region is indicated by caps colour-coded with $\omega^{2}$. The surface normal of the iso-surface element points in the direction of the positive enstrophy gradient. Different areas of the iso-surface are classified by either concave or convex areas, which is the shape observed when looking from the turbulent region towards the iso-surface. Examples of the respective surface types are indicated in figure $6(b)$. For the subsequent analysis, the local entrainment velocity is conditioned on the curvature of the local iso-surface element. Specifically, $v_{n}$ is conditioned on mean curvature, $H=\nabla \cdot \boldsymbol{n} / 2=\left(\kappa_{1}+\kappa_{2}\right) / 2$, and Gaussian curvature, $K=\kappa_{1} \kappa_{2}$, where $\boldsymbol{n}$ is the isosurface normal vector and $\kappa_{1}$ and $\kappa_{2}$ are the principal curvatures. Figure $6(b)$ shows the different iso-surface geometries in the $H-K$ plane. The zone $K>H^{2}$ in the $H-K$ plane implies complex curvatures, which do not occur for the investigated iso-surface.

We used a paraboloid fitting procedure to estimate Gaussian and mean curvature values of the triangular mesh of the enstrophy iso-surface. Curvatures of each vertex on the triangulated surface are estimated by a least-squares fitting of an osculating paraboloid to the vertex and its surrounding neighbours. Details about the procedure are given in Stokely \& Wu (1992) and Magid, Soldea \& Rivlin (2007), for example.

Figure 7 depicts joint p.d.f.s of mean and Gaussian curvatures $H$ and $K$ for the four flow configurations investigated (ZMS, jet $R e$ 2000, $R e$ 5000, $R e$ 8000). Contours are in logarithmic scale. All samples in the joint p.d.f. are below the parabola $K=H^{2}$, which separates real and complex curvature regions. It can be seen for all four cases that iso-probability contours surround the origin and decrease in magnitude for increasing curvature values. Areas of highest probability are shifted towards small negative mean curvature values, whereas the entire joint distribution is moderately skewed towards positive $H$ values. These features are most visible for the DNS data of the ZMS flow, which presumably reflects the slightly higher spatial resolution of the 
Zero mean shear Jet Re $2000 \quad$ Jet $\operatorname{Re} 5000 \quad$ Jet $\operatorname{Re} 8000$

\begin{tabular}{|c|c|c|c|c|}
\hline$\frac{\left\langle v_{n}\right\rangle}{u_{\eta}}$ & -0.15 & -0.26 & -0.50 & -0.52 \\
\hline$\frac{\left\langle v_{n}^{i n v}\right\rangle}{\left\langle v_{n}\right\rangle}$ & 0.37 & 0.39 & 0.40 & 0.35 \\
\hline$\frac{\left\langle v_{n}^{v i s}\right\rangle}{\left\langle v_{n}\right\rangle}$ & 0.63 & 0.61 & 0.60 & 0.65 \\
\hline$\frac{i n v_{1}}{\left\langle v_{n}\right\rangle}$ & 0.37 & 0.23 & 0.19 & 0.21 \\
\hline$\frac{i n v_{2}}{\left\langle v_{n}\right\rangle}$ & 0.00 & 0.20 & 0.18 & 0.16 \\
\hline$\frac{i n v_{3}}{\left\langle v_{n}\right\rangle}$ & 0.00 & -0.02 & 0.00 & -0.02 \\
\hline$\frac{i n v_{4}}{\left\langle v_{n}\right\rangle}$ & 0.00 & 0.00 & 0.00 & 0.00 \\
\hline$\frac{i n v_{5}}{\left\langle v_{n}\right\rangle}$ & 0.00 & 0.01 & 0.00 & 0.01 \\
\hline$\frac{i n v_{6}}{\left\langle v_{n}\right\rangle}$ & 0.00 & -0.03 & 0.03 & -0.01 \\
\hline$\frac{v i s_{1}}{\left\langle v_{n}\right\rangle}$ & 0.63 & 0.31 & 0.34 & 0.41 \\
\hline$\frac{v i s_{2}}{\left\langle v_{n}\right\rangle}$ & 0.00 & 0.31 & 0.27 & 0.24 \\
\hline$\frac{v i s_{3}}{\left\langle v_{n}\right\rangle}$ & 0.00 & 0.00 & 0.00 & 0.01 \\
\hline$\frac{v i s_{4}}{\left\langle v_{n}\right\rangle}$ & 0.00 & -0.01 & -0.01 & -0.01 \\
\hline
\end{tabular}

TABLE 2. Conditional mean values for $v_{n}$ normalized by $u_{\eta}$ as well as ratios of $v_{n}^{i n v}$ and $v_{n}^{v i s}$ and their components for the position at the interface, i.e. $\hat{x}_{n}=0$.

simulation. By and large, distributions of $H$ and $K$ seem unaffected by the different flow configurations.

In contrast, conditional averages of $v_{n}$ with respect to surface curvatures $H$ and $K$ show a considerable dependence on flow configurations, as shown in figure 8 . It is found that with increasing Taylor Reynolds number $R e_{\lambda}$, i.e. from ZMS with $R e_{\lambda}=50$ to jet $\operatorname{Re} 8000$ with $R e_{\lambda}=100$, the local entrainment velocity reaches higher negative values. This implies that, while $u_{\eta}$ seems to be the correct scaling parameter for $v_{n}$, i.e. $v_{n} / u_{\eta}=O(1)$, the precise ratio may depend on Reynolds number, at least for the moderate $R e$ investigated here. In addition, the decrease of the velocity from negative to positive $H$ values becomes more pronounced with higher $R e$. This trend will be discussed in more detail in $\$ 3.4$, figures 9-11. The results for the jet at $R e=5000$ and $R e=8000$ depict very similar distributions of $v_{n}$. A possible explanation is that these two configurations lie in the range of the so-called 'mixing 

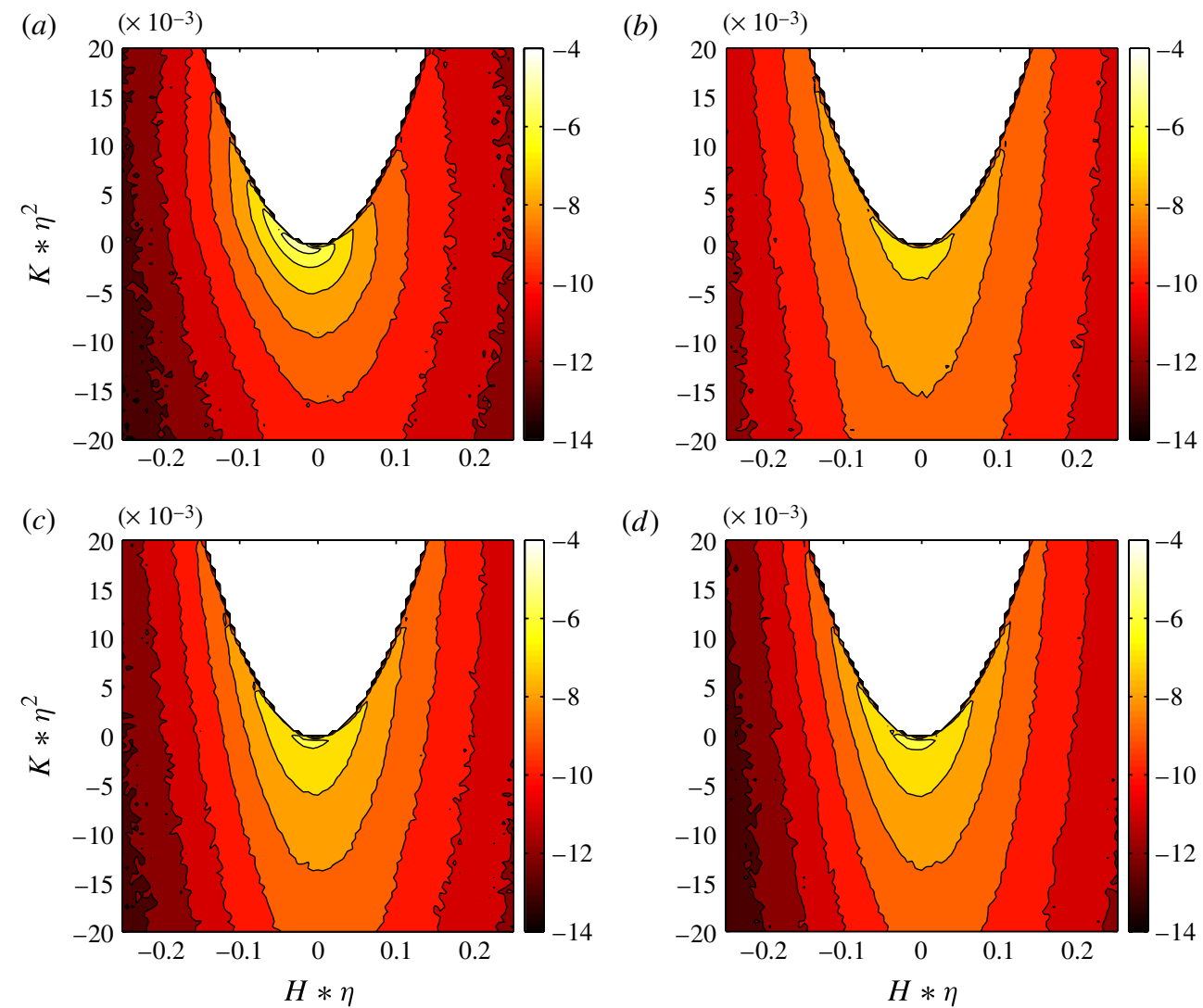

FIgURE 7. (Colour online) Conditional average of Gaussian and mean curvature $K$ and $H$ : (a) ZMS; (b) jet $\operatorname{Re} 2000$; (c) jet $\operatorname{Re} 5000$; (d) jet $\operatorname{Re} 8000$.

transition Reynolds number'. As discussed in Dimotakis (2005), flow dynamics of partly turbulent flows seem to change beyond a Taylor Reynolds number of $\sim 100$, resulting in a broader spectrum of eddying scales and a weaker Reynolds number dependence of various flow phenomena including turbulent entrainment. In short, we see a Reynolds number dependence of $v_{n}$ for the investigated flows, reflecting a possible effect of the increasing mean shear. Whether the trend continues for higher Reynolds numbers remains to be seen.

Some features of the distribution seem to be independent of the flow type. For all flow configurations, the local entrainment velocity is observed to depend on the local shape of the interface. In particular, $v_{n}$ tends towards values around zero for concave surface shapes $(H * \eta<0)$ and attains higher negative values in areas of convex shape $(H * \eta>0)$. From this it follows that in convex areas local entrainment of irrotational fluid into the turbulent flow region is higher than for convex shapes. Moreover, this finding is an indication that $v_{n}$ counteracts the convolution of the iso-surface, since convex shapes advance into the ambient fluid with a higher speed than concave shapes. Mean curvature $H$ appears to be the main parameter for the change of $v_{n}$.

\subsection{Decomposition of the local entrainment velocity}

In order to directly relate effects of mean shear on the local entrainment velocity, we present a decomposition of $v_{n}$ into conditional mean and fluctuating quantities as 

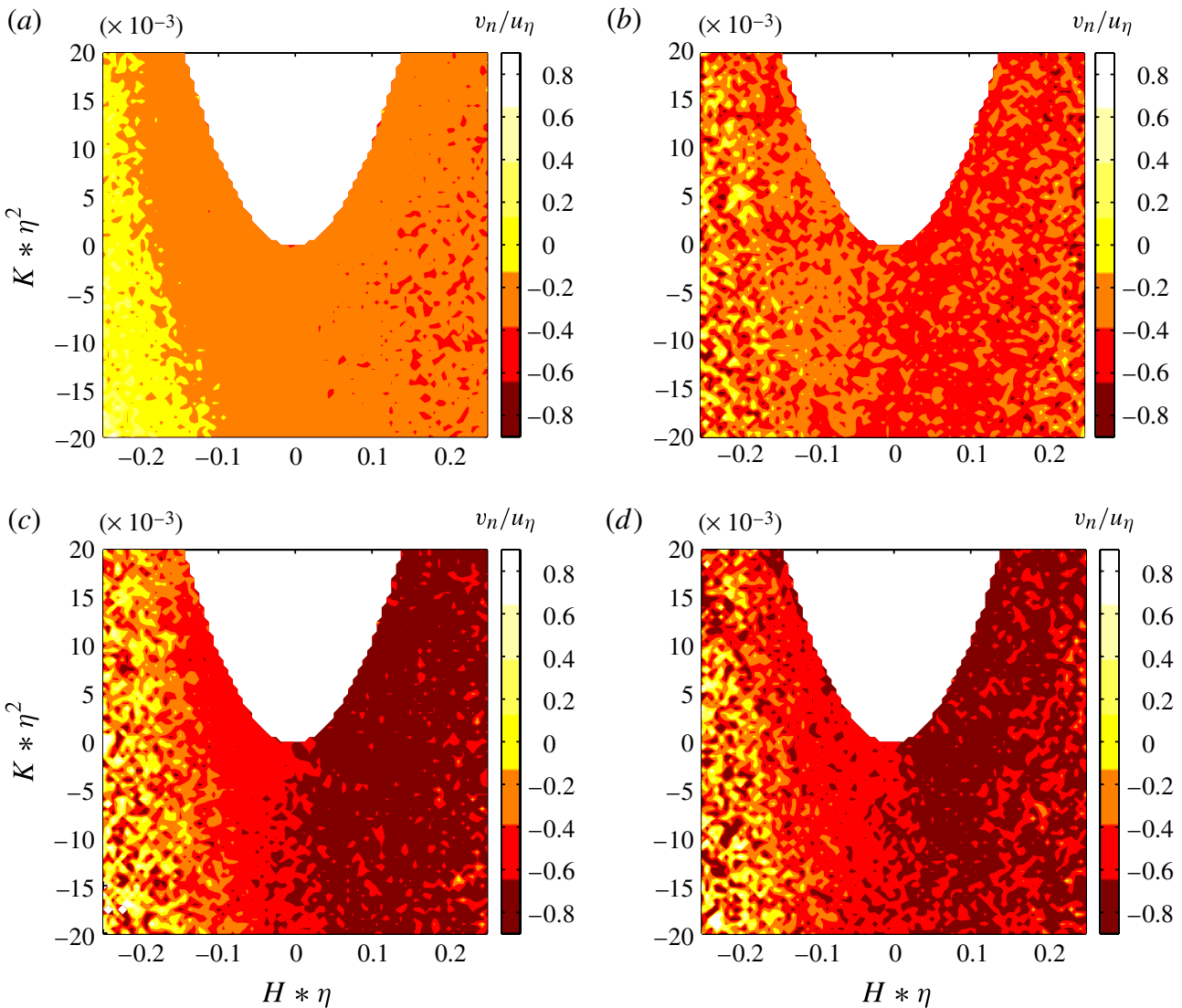

FIGURE 8. (Colour online) $v_{n}$ conditioned on Gaussian and mean curvature $K$ and $H$, normalized by $u_{\eta}:(a) \mathrm{ZMS}$; (b) jet $\operatorname{Re} 2000 ;(c)$ jet $\operatorname{Re} 5000 ;(d)$ jet $\operatorname{Re} 8000$.

described in (2.1). Inviscid and viscous contribution of the local entrainment velocity, $v_{n}^{\text {inv }}$ and $v_{n}^{\text {vis }}$, are divided into conditional averages and fluctuations of strain and vorticity. The resulting expression for $v_{n}^{i n v}$ reads

$$
\begin{aligned}
v_{n}^{i n v} & =-\frac{2 \omega_{i} \omega_{j} s_{i j}}{\left|\nabla \omega^{2}\right|} \\
& =-\frac{2\left(\omega_{i}^{\prime}+\Omega_{i}\right)\left(\omega_{j}^{\prime}+\Omega_{j}\right)\left(s_{i j}^{\prime}+S_{i j}\right)}{\left|\nabla \omega^{2}\right|} \\
& =-2 \frac{\omega_{i}^{\prime} \omega_{j}^{\prime} s_{i j}^{\prime}+\omega_{i}^{\prime} \omega_{j}^{\prime} S_{i j}+2 \omega_{i}^{\prime} \Omega_{j} s_{i j}^{\prime}+2 \omega_{i}^{\prime} \Omega_{j} S_{i j}+\Omega_{i} \Omega_{j} s_{i j}^{\prime}+\Omega_{i} \Omega_{j} S_{i j}}{\left|\nabla \omega^{2}\right|} .
\end{aligned}
$$

Taking the mean of the inviscid entrainment velocity, we obtain the following six terms:

$$
\begin{aligned}
\left\langle v_{n}^{i n v}\right\rangle= & -2\left[\left\langle\frac{\omega_{i}^{\prime} \omega_{j}^{\prime} s_{i j}^{\prime}}{\left|\nabla \omega^{2}\right|}\right\rangle+\left\langle\frac{\omega_{i}^{\prime} \omega_{j}^{\prime} S_{i j}}{\left|\nabla \omega^{2}\right|}\right\rangle+2\left\langle\frac{\omega_{i}^{\prime} \Omega_{j} s_{i j}^{\prime}}{\left|\nabla \omega^{2}\right|}\right\rangle\right. \\
& \left.+2\left\langle\frac{\omega_{i}^{\prime} \Omega_{j} S_{i j}}{\left|\nabla \omega^{2}\right|}\right\rangle+\left\langle\frac{\Omega_{i} \Omega_{j} s_{i j}^{\prime}}{\left|\nabla \omega^{2}\right|}\right\rangle+\left\langle\frac{\Omega_{i} \Omega_{j} S_{i j}}{\left|\nabla \omega^{2}\right|}\right\rangle\right] \\
= & i n v_{1}+i n v_{2}+i n v_{3}+i n v_{4}+i n v_{5}+i n v_{6} .
\end{aligned}
$$




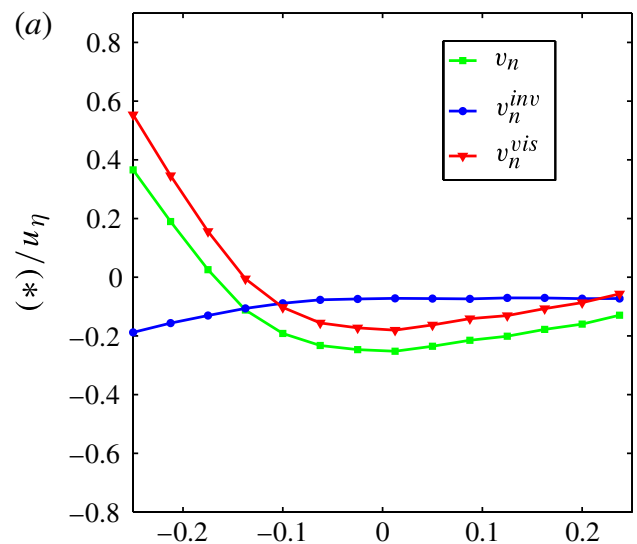

(b)

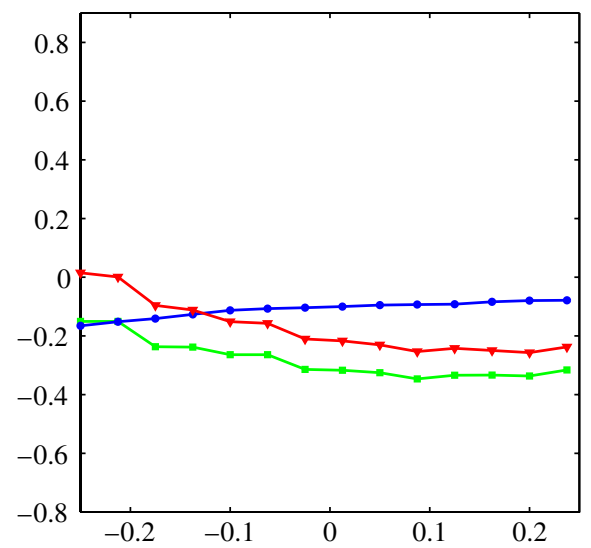

(c)

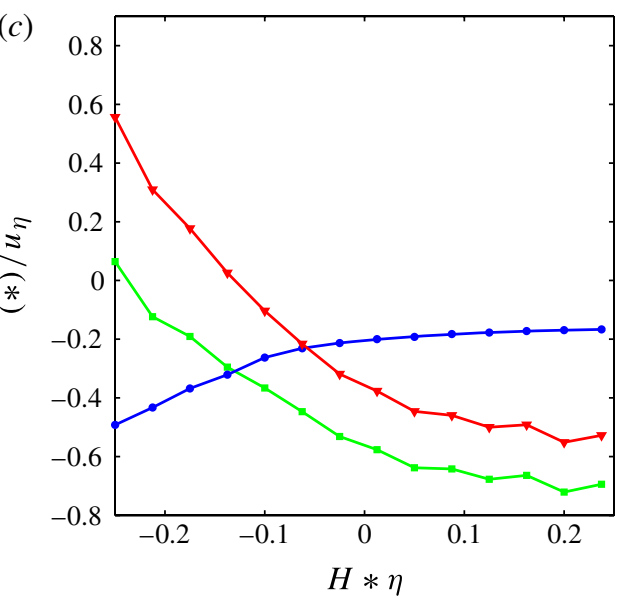

(d)

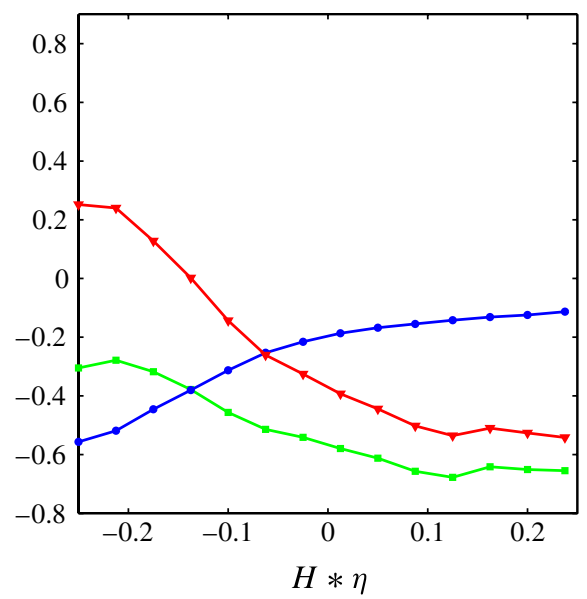

FIGURE 9. (Colour online) $v_{n}, v_{n}^{\text {inv }}$ and $v_{n}^{v i s}$ conditioned on mean curvature $H$, normalized by $u_{\eta}$ : (a) ZMS; (b) jet $\operatorname{Re} 2000 ;(c)$ jet $\operatorname{Re} 5000 ;(d)$ jet $\operatorname{Re} 8000$. Concave regions $(H * \eta<0)$, convex regions $(H * \eta>0)$.

The viscous component of the local entrainment velocity, $v_{n}^{v i s}$, can be decomposed as

$$
\begin{aligned}
v_{n}^{v i s} & =-\frac{2 v \omega_{i} \nabla^{2} \omega_{i}}{\left|\nabla \omega^{2}\right|} \\
& =-\frac{2 v\left(\omega_{i}^{\prime}+\Omega_{i}\right) \nabla^{2}\left(\omega_{i}^{\prime}+\Omega_{i}\right)}{\left|\nabla \omega^{2}\right|} \\
& =-2 v \frac{\omega_{i}^{\prime} \nabla^{2} \omega_{i}^{\prime}+\Omega_{i} \nabla^{2} \Omega_{i}+\Omega_{i} \nabla^{2} \omega_{i}^{\prime}+\omega_{i}^{\prime} \nabla^{2} \Omega_{i}}{\left|\nabla \omega^{2}\right|} .
\end{aligned}
$$

Taking the mean of the viscous entrainment velocity, we get

$$
\begin{aligned}
\left\langle v_{n}^{v i s}\right\rangle & =-2 v\left[\left\langle\frac{\omega_{i}^{\prime} \nabla^{2} \omega_{i}^{\prime}}{\left|\nabla \omega^{2}\right|}\right\rangle+\left\langle\frac{\Omega_{i} \nabla^{2} \Omega_{i}}{\left|\nabla \omega^{2}\right|}\right\rangle+\left\langle\frac{\Omega_{i} \nabla^{2} \omega_{i}^{\prime}}{\left|\nabla \omega^{2}\right|}\right\rangle+\left\langle\frac{\omega_{i}^{\prime} \nabla^{2} \Omega_{i}}{\left|\nabla \omega^{2}\right|}\right\rangle\right] \\
& =v i s_{1}+v i s_{2}+v i s_{3}+v i s_{4} .
\end{aligned}
$$


Table 2 shows conditional mean values for the local entrainment velocity as well as ratios of inviscid and viscous contributions and their components (equations (3.2) and (3.4)) for the position at the interface, i.e. $\hat{x}_{n}=0$. Consistent with the findings in $\S 3.3$, it can be seen that conditional averages of $v_{n}$ normalized by $u_{\eta}$ grow in magnitude from the case of ZMS to the case of jet $R e=8000$. Zero-mean-shear flow and jet $R e=2000$ have similar Taylor Reynolds numbers, i.e. $R e_{\lambda}=50$ in comparison to $R e_{\lambda}=60$, which indicates that not only the augmentation of the Reynolds number but also the effect of mean shear contributes to an enhanced local entrainment velocity.

Looking at the decomposition of the inviscid entrainment velocity for the three jet cases, we can see that in addition to the term comprising only fluctuations, $i n v_{1}=-2\left\langle\omega_{i}^{\prime} \omega_{j}^{\prime} s_{i j}^{\prime} /\left|\nabla \omega^{2}\right|\right\rangle$, the term consisting of vorticity fluctuations and mean strain, $i n v_{2}=-2\left\langle\omega_{i}^{\prime} \omega_{j}^{\prime} S_{i j} /\left|\nabla \omega^{2}\right|\right\rangle$, also has a significant influence on the inviscid entrainment component. The picture is similar for the viscous component, where we identify the term comprising only fluctuations, vis $=-2 v\left\langle\omega_{i}^{\prime} \nabla^{2} \omega_{i}^{\prime} /\left|\nabla \omega^{2}\right|\right\rangle$, and also the term comprising only mean values, vis $=-2 v\left\langle\Omega_{i} \nabla^{2} \Omega_{i} /\left|\nabla \omega^{2}\right|\right\rangle$, to be the dominating components of $v_{n}^{\text {vis }}$. This illustrates that for the viscous component too, mean shear seems to have a significant influence on the entrainment process. Interestingly, mean strain seems to be the influencing factor for $v_{n}^{i n v}$, whereas mean vorticity is of less importance for the inviscid component and shows an impact only on $v_{n}^{v i s}$. Furthermore, the influence of mean quantities appears to slightly decrease for higher Reynolds numbers. The ZMS case has by its very nature only a contribution of solely fluctuating components, i.e. $i n v_{1}=-2\left\langle\omega_{i}^{\prime} \omega_{j}^{\prime} s_{i j}^{\prime} /\left|\nabla \omega^{2}\right|\right\rangle$ and $v i s_{1}=-2 v\left\langle\omega_{i}^{\prime} \nabla^{2} \omega_{i}^{\prime} /\left|\nabla \omega^{2}\right|\right\rangle$. In conclusion, table 2 illustrates a direct effect of mean shear on small-scale processes of the local entrainment process resulting in an enhanced entrainment velocity with respect to the case of ZMS. The findings described are in accordance with the proposition of Corrsin \& Kistler (1954), who postulated that the local entrainment velocity depends on mean and fluctuating shear forces at the turbulent boundary.

In the following, we investigate the dependence of $v_{n}, v_{n}^{i n v}$ and $v_{n}^{v i s}$ on the local shape of the convoluted entrainment interface. The analysis focuses on the mean curvature, since it appears to be the governing parameter, as seen above in $\S 3.3$. Figure 9 shows the local entrainment velocity as well as its inviscid and viscous components conditioned on mean curvature. Looking at inviscid and viscous components of the local entrainment velocity, one can see that, independent of the curvature value, $v_{n}^{i n v}$ is on average negative for all four flow configurations and therefore always contributes to the outward spreading of the turbulent front. In contrast, $v_{n}^{\text {vis }}$ attains positive values for concave surface shapes, which implies that it even counteracts the entrainment process at those curvatures. In summary, we find for all flow configurations that, depending on the surface shape, different small-scale mechanisms control the local entrainment process. The viscous component of the local entrainment velocity related to viscous effects, $v \omega_{i} \nabla^{2} \omega_{i}$, governs local entrainment for convex shapes $(H * \eta>0)$ and the inviscid component related to vortex stretching, $\omega_{i} \omega_{j} s_{i j}$, does the same for concave shapes $(H * \eta<0)$. In other words, vortex stretching tries to convolute the interface, whereas viscous effects appear to flatten it. The characteristics described exist in more or less strong manifestations for all four flow configurations. Conversely, the local entrainment velocity of the ZMS flow attains on average positive values in the region of higher negative $H$ values, suggesting that isosurface elements of distinct concavity even tend to move towards the turbulent region. From the jet experiments, we see that mean shear adds to the outward spreading of the interface by shifting $v_{n}$ to purely negative values. 


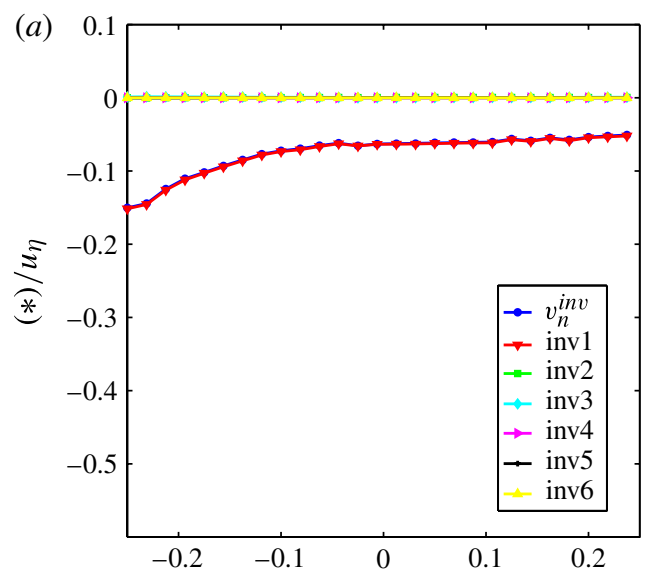

(b)
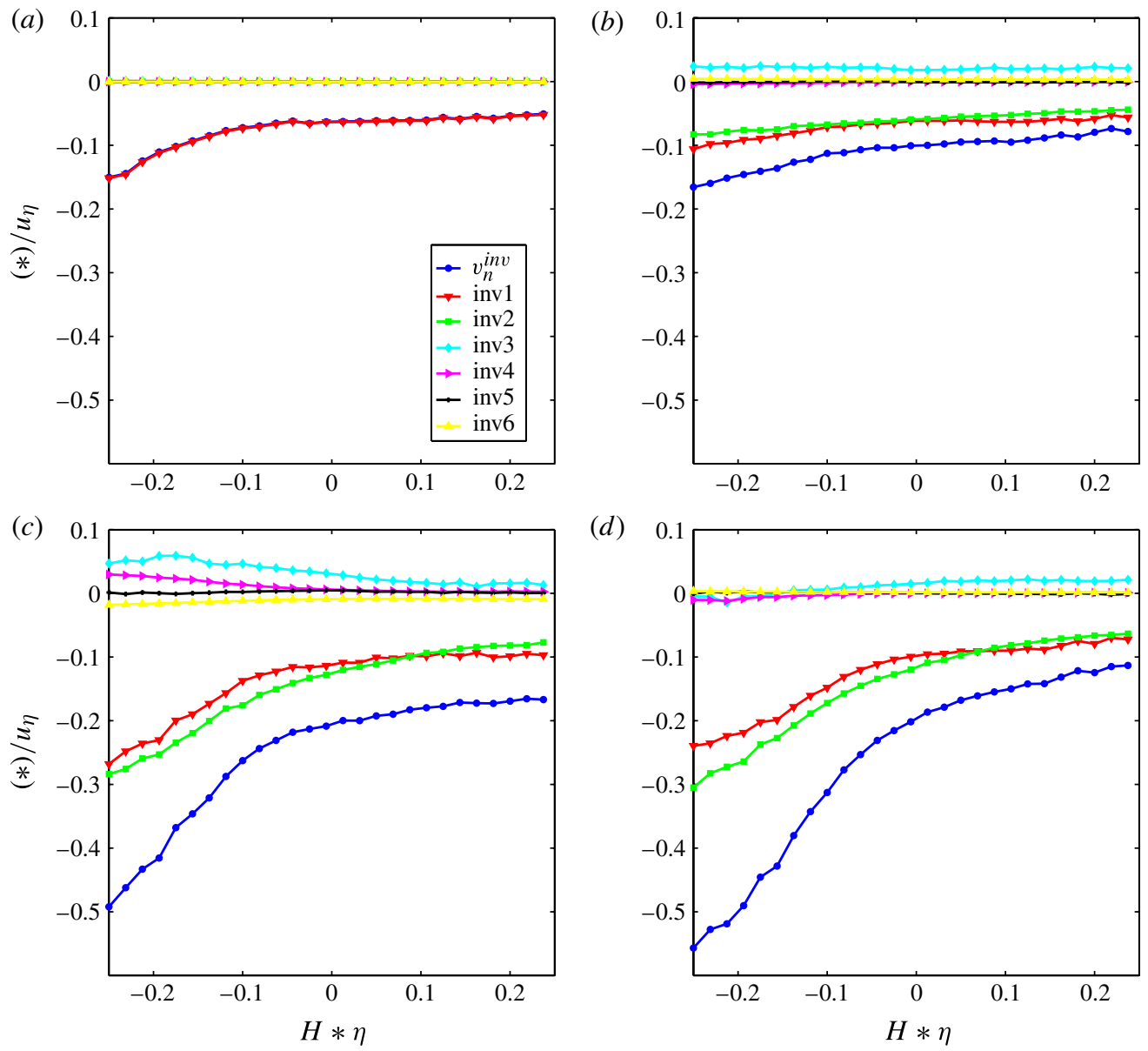

(d)

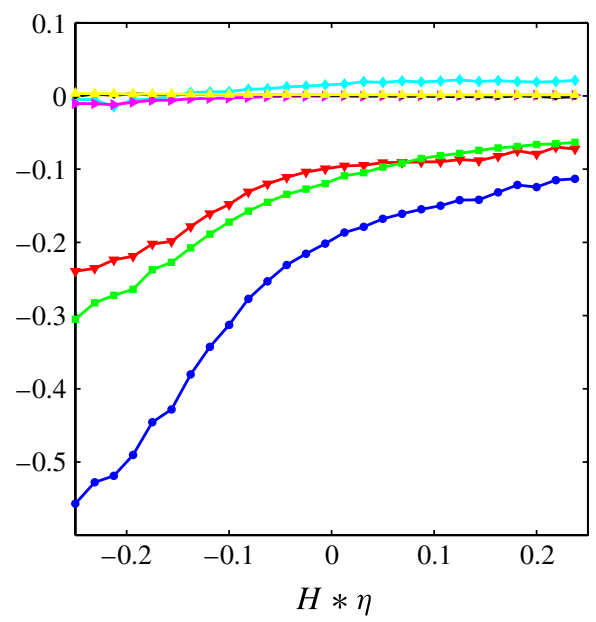

FIGURE 10. (Colour online) $v_{n}^{i n v}$ and its components conditioned on mean curvature $H$, normalized by $u_{\eta}$ : (a) ZMS; (b) jet $\operatorname{Re} 2000 ;(c)$ jet $R e$ 5000; $(d)$ jet $R e$ 8000. Concave regions $(H * \eta<0)$, convex regions $(H * \eta>0)$.

Looking at mean and fluctuating components of $v_{n}^{i n v}$ and $v_{n}^{v i s}$, the effect of mean quantities with respect to the surface shape becomes directly visible. Figure 10 depicts average profiles of $v_{n}^{i n v}$ and its components conditioned on mean curvature $H$. For the ZMS case, $v_{n}^{i n v}$ is equal to $i n v_{1}=-2\left\langle\omega_{i}^{\prime} \omega_{j}^{\prime} s_{i j}^{\prime} /\left|\nabla \omega^{2}\right|\right\rangle$, since mean values are zero for the flow. The jets show an additional influence of $i n v_{2}=-2\left\langle\omega_{i}^{\prime} \omega_{j}^{\prime} S_{i j} /\left|\nabla \omega^{2}\right|\right\rangle$ related to mean strain, which shifts the inviscid entrainment component to higher negative values, which supports the observation from figure 9. Furthermore, it is found that, independently of the curvature value, $i n v_{1}$ and $i n v_{2}$ contribute at similar proportions to the inviscid component of the local entrainment velocity.

The curvature effect is stronger for mean and fluctuating components of the viscous entrainment velocity. Figure 11 shows average profiles of $v_{n}^{v i s}$ and its components conditioned on mean curvature $H$, normalized by $u_{\eta}$. Again, the only non-zero component of the viscous entrainment velocity for the ZMS flow is $v i s_{1}$. The main components influencing the viscous entrainment process for jets are $v i s_{1}=-2 v\left\langle\omega_{i}^{\prime} \nabla^{2} \omega_{i}^{\prime} /\left|\nabla \omega^{2}\right|\right\rangle$ and $v i s_{2}=-2 v\left\langle\Omega_{i} \nabla^{2} \Omega_{i} /\left|\nabla \omega^{2}\right|\right\rangle$. Interestingly, the 


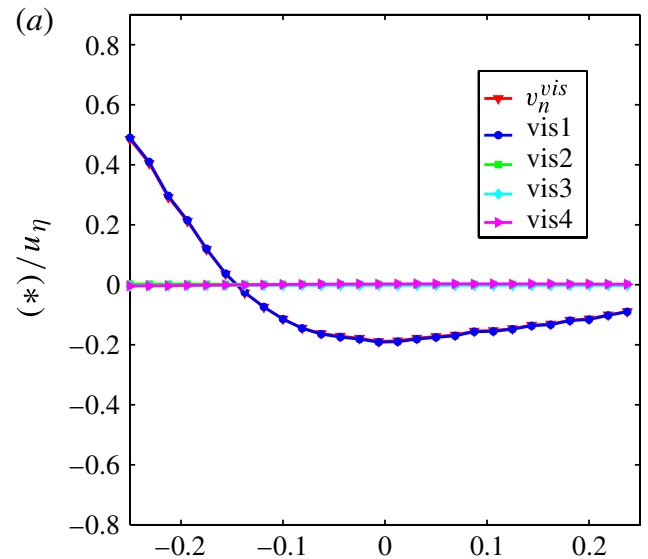

(b)
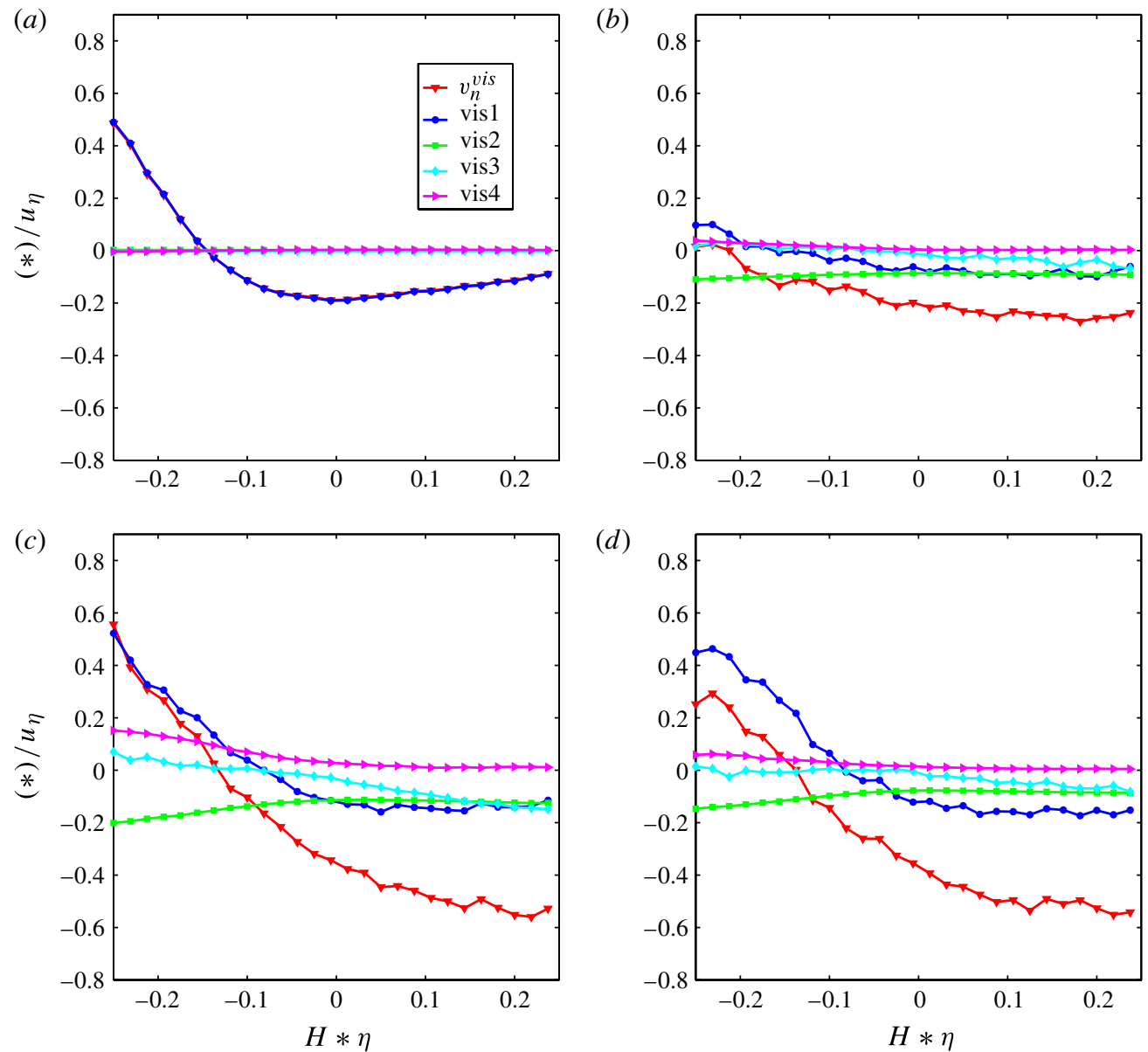

(d)

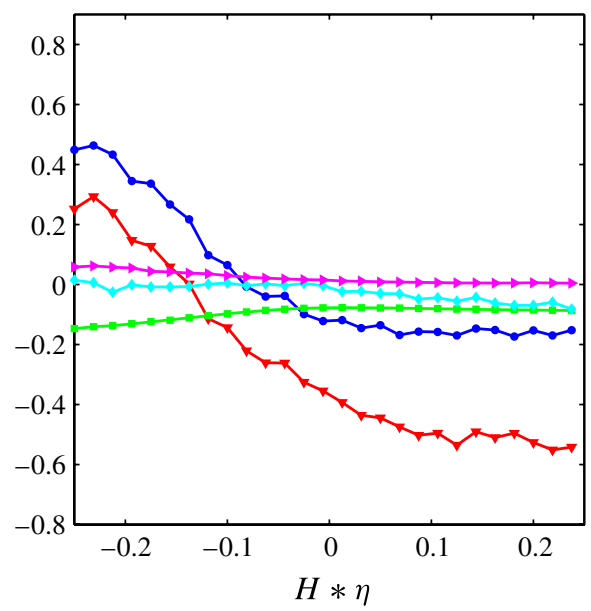

FIGURE 11. (Colour online) $v_{n}^{v i s}$ and its components conditioned on mean curvature $H$, normalized by $u_{\eta}$ : (a) ZMS; (b) jet $\operatorname{Re} 2000 ;(c)$ jet $\operatorname{Re} 5000 ;(d)$ jet $\operatorname{Re} 8000$. Concave regions $(H * \eta<0)$, convex regions $(H * \eta>0)$.

characteristic of the two terms differs considerably depending on the mean curvature. Component vis shows a nearly constant contribution to the viscous entrainment process independent of the curvature value. In contrast, component vis $s_{1}$ highly depends on the shape of the interface, adopting positive values for concave shapes $(H * \eta<0)$ and negative values for convex shapes $(H * \eta>0)$. From this we conclude that viscous effects due to vorticity fluctuations try to flatten or at least not to further convolute the interface. Figures 10 and 11 show that mean shear contributes to both the inviscid and the viscous entrainment velocity, shifting them to higher negative values, i.e. increasing the entrainment process at the interface.

\section{Conclusion}

This paper investigates effects of mean shear on the local turbulent entrainment process. Three-dimensional particle tracking velocimetry measurements of an axisymmetric jet are compared to the results of a DNS of a ZMS flow. Quality checks of the jet experiments show that the accuracy of the obtained data is sufficiently 
high to study small-scale mechanisms of the entrainment process, which are related to velocity derivatives.

In summary, three main effects of mean shear on the local entrainment process are demonstrated. First, investigating conditional enstrophy profiles relative to the interface, it is found that in a pseudo-Eulerian frame of reference, vorticity fluctuations as well as mean shear contribute to the vorticity jump at the boundary between irrotational and turbulent regions. Secondly, consistent with the postulate in Corrsin \& Kistler (1954), mean shear enhances the local entrainment velocity by contributing to its inviscid and viscous components. This reflects the influence of mean quantities on small-scale processes such as vortex stretching and viscous effects. Thirdly, mean shear causes entrainment in surface areas where vorticity fluctuations are counteracting the average interface movement into the non-turbulent flow region.

Two observations of the entrainment process were made independent of the flow type, which suggest that they possibly reflect universal features of the turbulent entrainment process. First, we found that during the first Kolmogorov times after passing the interface, the evolution of enstrophy along trajectories crossing the entrainment interface is almost exclusively governed by vorticity fluctuations, and that enstrophy values in the turbulent region are considerably smaller than in the pseudoEulerian frame of reference. A mapping between normal distance to the instantaneous interface versus conditional time along the trajectory showed that entraining particles tend to stay in close proximity to the entrainment interface, which explains the resulting lower average enstrophy values compared to the pseudo-Eulerian view. Second, we saw for all investigated flow configurations that local entrainment depends on the local shape of the interface, increasing for convex surface elements, looking from the turbulent region towards the convoluted boundary. Furthermore, depending on the surface shape, different small-scale mechanisms are dominant for the local entrainment process, i.e. viscous effects for convex shapes and vortex stretching for concave shapes.

The presented study is limited to a maximum Taylor Reynolds number of 100 . We confirm that $v_{n} / u_{\eta}=O(1)$ also for flows with mean shear. The precise ratio may depend on the Reynolds number, at least for the moderate $R e$ investigated here. The influence of mean quantities on inviscid and viscous components of the entrainment velocity appears to decrease slightly for higher Reynolds numbers. The question remains whether the trend continues for higher values of $R e_{\lambda}$.

Most studies so far have used an Eulerian description to investigate turbulent entrainment. Our results suggest that new insights into the entrainment process can be found if Eulerian and Lagrangian views are compared to each other.

\section{Acknowledgement}

We gratefully acknowledge the support of this work by the Georg Fischer Fund under grant ETH-14 10-2.

\section{REFERENCES}

Bisset, D. K., Hunt, J. C. R. \& Rogers, M. M. 2002 The turbulent/non-turbulent interface bounding a far wake. J. Fluid Mech. 451, 383-410.

Coles, D. 1962 Interfaces and intermittency in turbulent shear flow. Mécanique de la Turbulence, CNRS 108, 229-248.

CORRSin, S. \& Kistler, A. 1954 The free-stream boundaries of turbulent flows. NACA, TN-3133, TR-1244, pp. 1033-1064. 
Dahm, W. \& Dimotakis, P. 1990 Mixing at large Schmidt number in the self-similar far field of turbulent jets. J. Fluid Mech. 217, 299-330.

Dimotakis, P. 2005 Turbulent mixing. J. Fluid Mech. 37, 329-356.

Dopazo, C. 2006 Iso-scalar surfaces, mixing and reaction in turbulent flows. C. R. Mécanique 334, 483-492.

Dopazo, C., Martin, J. \& Hierro, J. 2007 Local geometry of isoscalar surfaces. Phys. Rev. E 76 (5), 1-11, 056316.

FerRey, P. \& AupoiX, B. 2006 Behaviour of turbulence models near a turbulent/non-turbulent interface revisited. Intl J. Heat Fluid Flow 27 (5), 831-837.

Ganapathisubramani, B., Lakshminarasimhan, K. \& Clemens, N. T. 2007 Determination of complete velocity gradient tensor by using cinematographic stereoscopic PIV in a turbulent jet. Exp. Fluids 42 (6), 923-939.

GOVInDARAJAN, R. 2004 Universal behaviour of entrainment due to coherent structures in turbulent shear flow. Phys. Rev. Lett. 88, 134503.

HinZE, J. O. 1975 Turbulence, 2nd edn. McGraw-Hill.

Holzner, M., Liberzon, A., Nikitin, N., Kinzelbach, W. \& Tsinober, A. 2007 Small-scale aspects of flows in proximity of the turbulent/non-turbulent interface. Phys. Fluids 19, 071702 .

Holzner, M., Liberzon, A., Nikitin, N., Lüthi, B., Kinzelbach, W. \& Tsinober, A. 2008 A Lagrangian investigation of the small-scale features of turbulent entrainment through particle tracking and direct numerical simulation. J. Fluid Mech. 598, 465-475.

Holzner, M. \& LÜthI, B. 2011 Laminar superlayer at the turbulence boundary. Phys. Rev. Lett. 106 (13), 1-4, 134503.

Holzner, M., Lüthi, B., Tsinober, A. \& Kinzelbach, W. 2009 Acceleration, pressure and related quantities in the proximity of the turbulent/non-turbulent interface. J. Fluid Mech. 639, $153-165$.

Hoyer, K., Holzner, M., LÜthi, B., Guala, M., Liberzon, A. \& Kinzelbach, W. 2005 3D scanning particle tracking velocimetry. Exp. Fluids 39 (5), 923-934.

Hunt, J. C. R., Eames, I., DA Silva, C. B. \& Westerweel, J. 2011 Interfaces and inhomogeneous turbulence. Phil. Trans. R. Soc. A 369 (1039), 811-832.

Hunt, J. C. R., Eames, I. \& Westerweel, J. 2006 Mechanics of inhomogeneous turbulence and interfacial layers. J. Fluid Mech. 554, 449-519.

Hunt, J. C. R., EAMES, I. \& Westerweel, J. 2008 Vortical interactions with interfacial shear layer. In Proceedings of IUTAM Conference on Computational Physics and New Perspectives in Turbulence, pp. 331-338. Springer.

Hussein, N. J. C., CAPp, S. P. \& GeORge, W. K. 1994 Velocity measurements in a high-Reynolds-number, momentum-conserving, axisymmetric, turbulent jet. J. Fluid Mech. 258, $31-75$.

Liberzon, A., LÜthi, B., Holzner, M., Tsinober, A., Ott, S., Berg, J. \& Mann, J. 2012 On the structure of acceleration in turbulence. Physica D 241, 208-215.

LÜthi, B., Tsinober, A. \& Kinzelbach, W. 2005 Lagrangian measurement of vorticity dynamics in turbulent flow. J. Fluid Mech. 528, 87-118.

MaAs, H. G., Gruen, A. \& Papantoniou, D. 1993 Particle tracking velocimetry in three-dimensional flows. Part 1. Photogrammetric determination of particle coordinates. Exp. Fluids 15, 133-146.

Magid, E., SoldeA, O. \& Rivlin, E. 2007 A comparison of Gaussian and mean curvature estimation methods on triangular meshes of range image data. Comput. Vis. Image Underst. 107, 139-159.

Mullin, J. A. \& DAHM, W. J. 2006 Dual-plane stereo particle image velocimetry measurements of velocity gradient tensor fields in turbulent shear flow. Part 2. Experimental results. Phys. Fluids 18, 035102.

Nikitin, N. 2006 Finite-difference method for incompressible Navier-Stokes equations in arbitrary orthogonal curvilinear coordinates. J. Comput. Phys. 217 (2), 759-781.

Philip, J. \& MARUsic, I. 2012 Large-scale eddies and their role in entrainment in turbulent jets and wakes. Phys. Fluids 24, 055108. 
Pope, S. B. 1988 The evolution of surfaces in turbulence. Intl J. Engng Sci. 26 (5), 445-469.

Ricou, F. \& Spalding, D. 1961 Measurements of entrainment by axisymmetric turbulent jets. J. Fluid Mech. 11, 21-32.

DA Silva, C. B. 2009 The behaviour of subgrid-scale models near the turbulent/nonturbulent interface in jets. Phys. Fluids 21, 081702.

DA Silva, C. B. \& PereirA, C. F. 2008 Invariants of the velocity-gradient, rate-of-strain, and rate-of-rotation tensors across the turbulent/nonturbulent interface in jets. Phys. Fluids 20 (5), $1-18,055101$.

DA Silva, C. B., Dos Reis, R. J. N. \& Pereira, J. C. F. 2011 The intense vorticity structures near the turbulent/non-turbulent interface in a jet. J. Fluid Mech. 685, 165-190.

DA Silva, C. B. \& TAVeirA, R. R. 2010 The thickness of the turbulent/nonturbulent interface is equal to the radius of the large vorticity structures near the edge of the shear layer. Phys. Fluids 22, 121702.

Sreenivasan, K. R., Ramshankar, R. \& Meneveau, C. 1989 Mixing, entrainment and fractal dimensions of surfaces in turbulent flows. Proc. R. Soc. Lond. A 421 (1860), 79-108.

StOKely, E. \& WU, S. Y. 1992 Surface parametrization and curvature measurement of arbitrary 3-D objects: five practical methods. IEEE Trans. Pattern Anal. Mach. Intell. 14 (8), 833-840.

Townsend, A. A. 1976 The Structure of Turbulent Shear Flow, 2nd edn. Cambridge University Press.

Tritton, D. J. 1988 Physical Fluid Dynamics, 2nd edn. Clarendon.

Tsinober, A. 2009 An Informal Conceptual Introduction to Turbulence, 2nd edn. Springer.

WesterweEl, J. 2005 Mechanics of the turbulent-nonturbulent interface of a jet. Phys. Rev. Lett. 95, 174501.

Westerweel, J., Fukushima, C., Pedersen, J. \& Hunt, J. 2009 Momentum and scalar transport at the turbulent/non-turbulent interface of a jet. J. Fluid Mech. 631, 199-230.

WillnefF, J. \& GRUEN, A. 2002 A new spatio-temporal matching algorithm for 3D-particle tracking velocimetry. In The 9th International Symposium on Transport Phenomena and Dynamics of Rotating Machinery, Honolulu, Hawaii, USA.

Wolf, M., Lüthi, B., Holzner, M., Krug, D., Kinzelbach, W. \& Tsinober, A. 2012 Investigations on the local entrainment velocity in a turbulent jet. Phys. Fluids 24 (10), 105110 Attached publisher's note: Erratum: 'Investigations on the local entrainment velocity in a turbulent jet' [Phys. Fluids 24, 105110 (2012)], Phys. Fluids 25, 019901 (2013).

WygnAnski, I. \& Fiedler, H. 1969 Some measurements in the self-preserving jet. J. Fluid Mech. 38 (3), 577-612.

Xu, G. \& Antonia, R. A. 2002 Effect of different initial conditions on a turbulent round free jet. Exp. Fluids 33, 677-683. 